\title{
Nonlinear Filtering Methods in INS/GPS Alignment
}

\author{
T. Numajima, N. Asaoka, M. Oiwa \\ and S. Sugimoto \\ Department of Electrical and Electronic Engineering, \\ Ritsumeikan University \\ e-mail: sugimoto@se.ritsumei.ac.jp
}

\begin{abstract}
In this paper, we propose a new method for landvehicle In-Motion Alignment by integrating Inertial Navigation System (INS) and the transfer Differential GPS (DGPS). Our aim in this work is to integrate the advantages of these systems and to develop the navigation system that does not require initial attitudes information of the vehicle by applying recursive nonlinear filterling techniques.
\end{abstract}

\section{Introduction}

In the case of our previously proposed navigation system [1], the initialization of INS navigation states should be completed prior to vehicle motion. By stopping the vehicle at the start point, these initial states can be computed by integrating INS data with static navigation data such as the velocity $0(\mathrm{ft} / \mathrm{s})$ and the initial position obtained from DGPS. However this initialization method usually requires 5 - 10 minutes and thus the vehicle must be stopped. And it is common that there is not enough time to stop at the start point. Thus, for reducing the initial alignment time, developing the In-Motion Alignment algorithm is desired.

Many research activities have focused on the problem of INS alignment [2]. In the case of small initial error angle of INS, the problem is often solved on the basis of a linearized description of INS error equations. Kalman filtering is a popular tool in handling estimation these of problems, but its optimality heavily depends on linearity. In a number of cases INS is often to be aligned under the conditions that the initial error angles are comparatively large which makes it necessary to take account of nonlinear character of the problem [3]. And so, we present applying nonlinear filtering to INS alignment problem. In the nonlinear filtering method, the conditional expectation of the variable to be estimated are estimated by means of Monte-Carlo methods which are based on the Law of Large Numbers.

For the INS error model of the proposed algorithm, the large azimuth error model [4], which formulates INS error (position, velocity, attitude) equations for large initial heading error and sensor error [2], is adopted with suitable modification for our coordinate system. And the large azimuth error model is not approximated the first order.

\section{Coodinate Systems}

To integrate the navigation systems, it is important to consider the coordinate systems that the navigation systems or included sensors refer to. This section defines the coordinate frames used in this paper and represents the angular relationship between them.

The coordinate frames are defined as follows:

i) The $E$ frame $\left(X_{E}, Y_{E}, Z_{E}\right)$ is the right-handed earth fixed coordinate frame. It has origin at the center of the earth; the $Z_{E \text {-axis is directed }}$ toward the North Pole; the $X_{E^{-}}$and $Y_{E \text {-axes }}$ are located in the equatorial plane, whereby the $X_{E}$-axis is directed toward the Greenwich Meridian. It is used for the definition of position location such as latitude and longitude.

ii) The $L$ frame $\left(X_{L}, Y_{L}, Z_{L}\right)$ is the right-handed locally level coordinate frame. The $X_{L^{-}}$and $Y_{L}$-axes are directed toward local north and east respectively; $Z_{L}$-axis is downward vertical at the local earth surface referenced position location. It is used for defining the angular orientation of the local vertical in the $E$ frame. 
iii) The $C$ frame $\left(X_{C}, Y_{C}, Z_{C}\right)$ is the right-handed computer frame that is defined by rotating the $L$ frame around negative $Z_{L}$-axis by the "wander angle" $\alpha$, the $Y_{C}$-axis is directed toward the negative $Y_{L}$-axis and the $Z_{C}$-axis is directed toward the negative $Z_{L}$-axis (upward vertical). It is used for integrating acceleration into velocity, and used as the reference for describing the strapdown sensor coordinate frame orientation.

iv) The $B\left(X_{B}, Y_{B}, Z_{B}\right)$ frame is the strapdown inertial sensor coordinate frame (body frame). The $X_{B}$-axis is directed toward the head of the vehicle; the $Y_{B}$-axis is the right-hand of the vehicle; the $Z_{B}$-axis is downward vertical to the $X_{B}-Y_{B}$ plane. The frame is fixed on the vehicle and rotates with the motion of the vehicle.

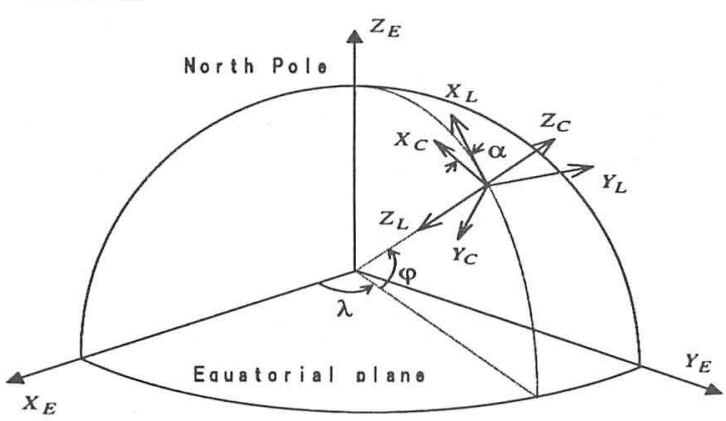

Fig. 1: The $E, L$ and $C$ frames

Fig. 1 shows the spatial image of the $E, L$ and $C$ frames, where $\lambda$ represents the geodetic longitude and $\varphi$ represents the latitude.

In inertial computations, three components of acceleration sensed with respect to the $B$ frame have to be transformed onto the $C$ frame. The velocity and position of the vehicle are then computed with respect to the $C$ frame. Such a transformation is known as an Euler angle transformation.

\section{Error model for INS}

Error can develop in the navigation system state through a variety of source. The initial values for the computed state will never exactly equal the actual navigation state. Therefore it is important to understand the dynamic behavior of the navigation-system error. In this section navigation system error analysis is presented. In the navigation of a land-vehicle by INS/DGPS integration, we assume that initialization error about azimuth error is large. Therefore, we adopt large azimuth error model [1] with suitable modification for our coordinate system. The nominal error state is defined to be position, velocity, and attitude errors.

\section{1 position error model}

The direction-cosine matrix $T_{E}^{C}$ is described as follows

$$
T_{E}^{C}=T_{L}^{C} T_{E}^{L}
$$

Actually, the computed matrices $\overline{T_{L}^{C}}$ and $\overline{T_{E}^{L}}$ which contains errors $\delta T_{L}^{C}$ and $\delta T_{E}^{L}$, respectively, such that the computed matrix $\frac{\bar{L}}{T_{E}^{C}}$ is given by

$$
\begin{aligned}
\overline{T_{E}^{C}} & =\left(T_{L}^{C}+\delta T_{L}^{C}\right)\left(T_{E}^{L}+\delta T_{E}^{L}\right) \\
& =\left(T_{L}^{C}+\delta T_{L}^{C}\right)\left[I-\left(\delta \boldsymbol{r}_{C} \times\right)\right] T_{E}^{L},
\end{aligned}
$$

where we can show the relation $\overline{T_{E}^{L}}=[I-$ $\left.\left(\delta \boldsymbol{r}_{C} \times\right)\right] T_{E}^{L}$ on the basis of assumption that horizontal angular position errors $\delta r_{C, x}, \delta r_{C, y}$ are small. Also, $(\boldsymbol{a} \times)$ for $3 \times 1$ vector $\boldsymbol{a}=\left[a_{x}, a_{y}\right.$, $\left.a_{z}\right]^{\mathrm{T}}$, denotes the skew-symmetric matrix defined by

$$
(a \times) \equiv\left[\begin{array}{ccc}
0 & -a_{z} & a_{y} \\
a_{z} & 0 & -a_{x} \\
-a_{y} & a_{x} & 0
\end{array}\right] .
$$

Thus the error $\delta T_{E}^{C}$ is described by

$$
\begin{aligned}
\delta T_{E}^{C} & \equiv \overline{T_{L}^{C}} \overline{T_{E}^{L}}-T_{L}^{C} T_{E}^{L} \\
& =\left\{\delta T_{L}^{C}\left[\boldsymbol{I}-\left(\delta r_{L} \times\right)\right]-T_{L}^{C}\left(\delta r_{L} \times\right)\right\} T_{L}^{C} T_{E}^{L},
\end{aligned}
$$

where $\delta T_{L}^{C}$ is obtained by

$$
\begin{aligned}
\delta T_{L}^{C}= & {\left[\begin{array}{ccc}
\cos (\alpha+\delta \alpha) & \sin (\alpha+\delta \alpha) & 0 \\
-\sin (\alpha+\delta \alpha) & \cos (\alpha+\delta \alpha) & 0 \\
0 & 0 & -1
\end{array}\right] } \\
& -\left[\begin{array}{ccc}
\cos \alpha & \sin \alpha & 0 \\
-\sin \alpha & \cos \alpha & 0 \\
0 & 0 & -1
\end{array}\right] .
\end{aligned}
$$

From Eqs. (4) and (5) and by the first-order approximation, the position error can be written by

$$
\delta T_{E}^{C}=\mathcal{R} T_{E}^{L} .
$$

$\mathcal{R}$ is defined as the position error matrix [1] as

$$
\mathcal{R} \equiv\left[\begin{array}{ccc}
\delta \cos \alpha & \delta \sin \alpha & \delta r_{C, y} \cos \delta \alpha-\delta r_{C, x} \sin \delta \alpha \\
-\delta \sin \alpha & \delta \cos \alpha & -\delta r_{C, x} \cos \delta \alpha-\delta r_{C, y} \sin \delta \alpha \\
-\delta r_{L, y} & \delta r_{L, x} & 0
\end{array}\right]
$$


where

$$
\begin{aligned}
& \delta \sin \alpha \equiv \sin (\alpha+\delta \alpha)-\sin \alpha \\
& \delta \cos \alpha \equiv \cos (\alpha+\delta \alpha)-\cos \alpha .
\end{aligned}
$$

According to [1]

$$
\begin{aligned}
\dot{\mathcal{R}}= & \mathcal{R}\left(\omega_{E / L}^{L} \times\right)+\left(\omega_{E / C}^{C} \times\right) T_{L}^{C} \\
& -\left(\overline{\omega_{E / C}^{C}} \times\right)\left(T_{L}^{C}+\mathcal{R}\right),
\end{aligned}
$$

where the dot above a letter denotes differentiation with respect to time, the vector $\omega_{E / L}^{L}$ is the rotation rate of the $L$ frame with respect to the $E$ frame in the $L$ frame coordinate system and also the vector $\boldsymbol{\omega}_{E / C}^{C}$ is similarly denoted.

Eq. (10) gives the position error $\left(: \delta r_{C, x}, \delta r_{C, y}\right)$ equations in INS navigation error, and also gives the relations of state variables: the wander angle error, $\sin \delta \alpha$ and $(\cos \delta \alpha-1)$.

\section{2 velocity error model}

The velocity error $\delta v_{C}$ is represented as a perturbation as follows

$$
\bar{v}_{C}=v_{C}+\delta v_{C} .
$$

The velocity navigation state $\boldsymbol{v}_{C}$ equation is represented as follows

$$
\dot{v}_{C}=\boldsymbol{f}_{C}-\left(\rho_{C}+\boldsymbol{\Omega}_{C}\right) \times \boldsymbol{v}_{C}-\boldsymbol{g}_{C},
$$

where $\boldsymbol{f}_{C}$ is the nongravitational specific force vector in the $C$ frame, $\rho_{C}$ is the vehicle angular rate with respect to the $E$ frame and $B \Omega_{C}$ is the eath rate. The specific force is proportional to the inertial acceleration of the system due to all forces except gravity, which is measured by an accelerometer. $g_{C}$ is the gravity vector considered positive toward the center of the earth in the $C$ frame. The computed specific force and gravity which contain errors are

$$
\begin{aligned}
& \overline{\boldsymbol{f}}_{C}=\left[\boldsymbol{I}-\left(\delta \underline{\boldsymbol{r}}_{C} \times\right)\right] \boldsymbol{f}_{C}+\delta \boldsymbol{b}_{C}, \\
& \overline{\boldsymbol{g}}_{C}=\boldsymbol{g}_{C}+\delta \boldsymbol{g}_{C},
\end{aligned}
$$

where $\delta b_{C}$ is the inertial sensor error. From Eqs. (11), (12), (13), the velocity error equation becomes

$$
\begin{array}{r}
\delta \underline{\dot{v}}_{C}=\delta \boldsymbol{b}_{C}+\boldsymbol{f}_{C} \times \delta \boldsymbol{\theta}_{C}+\boldsymbol{v}_{C} \times\left(\delta \boldsymbol{\rho}_{C}+2 \delta \boldsymbol{\Omega}_{C}\right)- \\
\left(\boldsymbol{\rho}_{C}+2 \delta \boldsymbol{\Omega}_{C}\right)-\left(\boldsymbol{\rho}_{C}+2 \delta \boldsymbol{\Omega}_{C}\right) \times \delta \boldsymbol{v}_{C}+\delta \boldsymbol{g}_{C},
\end{array}
$$

where $\delta \theta_{C} \equiv\left[\delta \theta_{C, x}, \delta \theta_{C, y}, \delta \theta_{C, z}\right]^{\mathrm{T}}$ is the attitude error. Eq. (14) gives velocity error models $\left(: \delta v_{C, x}\right.$, $\left.\delta v_{C, y}, \delta v_{C, z}\right)$.

\section{3 attitude error model}

The attitude error is defined as a tilt error. The error is associated with the transition matrix error $\delta T_{B}^{C}$ caused by the attitude error, $\delta \theta_{C}$. The computed matrix $\overline{T_{B}^{C}}$ which contains error is given by

$$
\overline{T_{B}^{C}}=\left[\boldsymbol{I}-\left(\delta \boldsymbol{\theta}_{C} \times\right)\right] T_{B}^{C} .
$$

Thus, we have

$$
\begin{aligned}
\delta T_{B}^{C} & =\bar{T}_{B}^{C}-T_{B}^{C}, \\
& =-\left(\delta \theta_{C} \times\right) T_{B}^{C} .
\end{aligned}
$$

Then $\dot{T}_{B}^{C}$ can be expressed by using the skewsymmetric matrix $\left(\omega_{B / C}^{C} \times\right)$ which means the relative angular velocity to the $C$ frame from the $B$ frame, namely

$$
\dot{T}_{B}^{C}=-\left(\omega_{B / C}^{C} \times\right) T_{B}^{C}
$$

From Eqs. (16) and (17), $\delta \dot{T}_{B}^{C}$ is obtained by

$$
\begin{aligned}
\delta \dot{T}_{B}^{C} & =-\left(\delta \dot{\boldsymbol{\theta}}_{C} \times\right) T_{B}^{C}-\left(\delta \boldsymbol{\theta}_{C} \times\right) \dot{T}_{B}^{C}, \\
& =-\left[(\delta \dot{\boldsymbol{\theta}} \times) T_{B}^{C}-\left(\delta \boldsymbol{\theta}_{C} \times\right)\left(\omega_{B / C}^{C} \times\right)\right] T_{B}^{C} .
\end{aligned}
$$

Also from Eqs. (15), (16) and (17) $\delta \dot{T}_{B}^{C}$ can be rewritten by

$$
\begin{aligned}
\delta \dot{T}_{B}^{C} & =-\left(\overline{\boldsymbol{\omega}_{B / C}^{C}} \times\right) \overline{T_{B}^{C}}+\left(\boldsymbol{\omega}_{B / C}^{C} \times\right) T_{B}^{C} \\
& =\left(\boldsymbol{\omega}_{B / C}^{C} \times\right)\left[\boldsymbol{I}-\left(\delta \boldsymbol{\theta}_{C} \times\right)\right] T_{B}^{C}+\left(\boldsymbol{\omega}_{B / C}^{C} \times\right) T_{B}^{C} .
\end{aligned}
$$

From Eqs. (18) and (19), we have

$$
\begin{aligned}
\left(\delta \dot{\boldsymbol{\theta}}_{C} \times\right)= & \left(\delta \boldsymbol{\theta}_{C} \times\right)\left(\boldsymbol{\omega}_{B / C}^{C} \times\right)-\left(\boldsymbol{\omega}_{B / C}^{C} \times\right)\left(\delta \boldsymbol{\theta}_{C} \times\right) \\
& +\left(\delta \boldsymbol{\omega}_{B / C^{B}}^{B}\right),
\end{aligned}
$$

namely,

$$
\delta \dot{\boldsymbol{\theta}}_{C}=\delta \boldsymbol{\theta}_{C} \times \omega_{B / C}^{C}+\left(\bar{\omega}_{B / C}^{C}-\omega_{B / C}^{C}\right) .
$$

In Eq. (21), the measurement data $\bar{\omega}_{B / C}^{C}$ is expressed by

$$
\bar{\omega}_{B / C}^{C}=\bar{\omega}_{I / C}^{C}-\bar{T}_{B}^{C}\left(\omega_{I / B}^{B}+d_{B}\right),
$$


where $d_{B}$ denotes the gyro drift in the computed value of the rotation vector. From Eqs. (21), (22), we have the following attitude error $\left(: \delta \theta_{C, x}, \delta \theta_{C, y}\right.$, $\left.\delta \theta_{C, z s}\right)$ equation as

$$
\delta \theta_{C}=\delta \omega_{E / C}^{C}+\omega_{I / E}^{C} \times \delta r_{C}+\delta \theta_{C} \times \omega_{I / C}^{C}+d_{C} .
$$

\section{4 sensor error model}

For the error models of inertial sensors in this work, they are modeled as the first order Markov processes,

$$
\begin{aligned}
& \dot{b}_{B}(t)=-\frac{1}{\tau_{b}} b_{B}(t)+u_{b, B}(t), \\
& \dot{d}_{B}(t)=-\frac{1}{\tau_{d}} d_{B}(t)+u_{d, B}(t),
\end{aligned}
$$

where $\boldsymbol{b}_{B}=\left[b_{B, x}, b_{B, y}, b_{B, z}\right]^{\mathrm{T}}$ is accelerometer bias, $\boldsymbol{d}_{B}=\left[d_{B, x}, d_{B, y}, d_{B, z}\right]^{\mathrm{T}}$ is gyro bias. Also $\tau_{b}$ and $\tau_{d}$ are the correlation time constants and $\boldsymbol{u}_{b, B}(t)$, $u_{d, B}(t)$ are zero mean Gaussian white noise processes.

\section{State Model}

The first step towards implementing the navigation filter is to define a system model which describes adequately the system dynamics. Using the coordinate systems defined in section 2 , we can now develop a set of nonlinear differential equations describing the propagation of INS errors in the $C$ frame navigation equations.

The states of the system to be estimated are thus described by the state vector $x$ as follows:

$$
\begin{aligned}
\boldsymbol{x}=[ & {\left[r_{x}, \delta r_{y}, \delta v_{x}, \delta v_{y}, \delta \theta_{x}, \delta \theta_{y}, \delta \sin \alpha,\right.} \\
& \left.(\delta \cos \alpha-1), \delta h, \delta v_{z}, b_{x}, b_{y}, b_{z}, d_{x}, d_{y}, d_{z}\right]^{\mathrm{T}}
\end{aligned}
$$

The description of the components is listed in Table 1.

The following dynamic model is then utilized:

$$
\dot{x}(t)=F(x(t), t)+\boldsymbol{\eta}(t),
$$

\begin{tabular}{|c|c|c|}
\hline No. & Symbol & Error state \\
\hline 1 & $\delta r_{x}$ & $X_{C}$-axis error in relative position \\
\hline 2 & $\delta r_{y}$ & $Y_{C}$-axis error in relative position \\
\hline 3 & $\delta v_{x}$ & $X_{C^{-}}$axis velocity error \\
\hline 4 & $\delta v_{y}$ & $Y_{C}$-axis velocity error \\
\hline 5 & $\delta \theta_{x}$ & $X_{C}$-axis tilt error \\
\hline 6 & $\delta \theta_{y}$ & $Y_{C^{-}}$axis tilt error \\
\hline 7 & $\sin \delta \alpha$ & sine of wander angle error \\
\hline 8 & $1-\cos \delta \alpha$ & $1-($ cosine of wander angle error $)$ \\
\hline 9 & $\delta h$ & $Z_{C}$-axis altitude error \\
\hline 10 & $\delta v_{z}$ & $Z_{C^{-}}$axis velocity error \\
\hline 11 & $b_{x}$ & $X_{B}$-axis accelerometer bias \\
\hline 12 & $b_{y}$ & $Y_{B}$-axis accelerometer bias \\
\hline 13 & $b_{z}$ & $Z_{B}$-axis accelerometer bias \\
\hline 14 & $d_{x}$ & $X_{B}$-axis gyro bias \\
\hline 15 & $d_{y}$ & $Y_{B}$-axis gyro bias \\
\hline 16 & $d_{z}$ & $Z_{B^{-} \text {-axis gyro bias }}$ \\
\hline
\end{tabular}

where $F(t)$ is the $16 \times 16$ time-varying matrix and the process noise $\boldsymbol{\eta}(t)$ is assumed to be mutually
Table. 1: List of states

independent zero mean Gaussian white noise with covariance matrix $N$ :

$$
E\left[\boldsymbol{\eta}(t) \boldsymbol{\eta}^{\mathrm{T}}(t)\right]=N(t) \delta(t)
$$

$(\delta(t)$ : Dirac's $\delta$ - function).

By discretizing the state equation (26), we have

$$
\boldsymbol{x}(k+1)=\boldsymbol{x}(k)+\boldsymbol{F}(\boldsymbol{x}(k), k) \Delta t+\boldsymbol{w}(k)
$$

where

$$
\begin{aligned}
& \boldsymbol{x}(k)=\left[\delta r_{x}(k \Delta t), \delta r_{x}(k \Delta t), \ldots, d_{z}(k \Delta t)\right]^{\mathrm{T}}, \\
& E\left[\boldsymbol{w}(k) \boldsymbol{w}^{\mathrm{T}}(j)\right]=Q(k) \delta_{k j},
\end{aligned}
$$

and, $\Delta t$ is a sampling interval for updating the measurement data.

\section{1 measurement equation}

In INS/DGPS integration system, the measurements are the differences between DGPS and INS positions, and velocities as well.

By the definition of $\delta r_{x}, \delta r_{y}$ and $\delta h$, the INSindicated positions at time $k$ are modeled as

$$
\begin{aligned}
& r_{x}^{i}(k)=r_{x}^{t}(k)+\delta r_{x}(k), \\
& r_{y}^{i}(k)=r_{y}^{t}(k)+\delta r_{y}(k), \\
& h^{i}(k)=h^{t}(k)+\delta h(k),
\end{aligned}
$$


where the superscripts $i$ and $t$ mean inertial and true respectively. The DGPS position indications are modeled as the true positions corrupted by mutually independent Gaussian white noises $\left\{n_{j}^{g}(k)\right\}$ with zero mean and diagonal covariance matrix $R^{g}(k)$ :

$$
\begin{aligned}
& r_{x}^{g}(k)=r_{x}^{t}(k)-n_{1}^{g}(k), \\
& r_{y}^{g}(k)=r_{y}^{t}(k)-n_{2}^{g}(k), \\
& h^{g}(k)=h^{t}(k)-n_{3}^{g}(k),
\end{aligned}
$$

where the superscript $g$ means DGPS. From Eqs. (31) and (32), the measurement models for position errors in consideration of the large azimuth error are given by

$$
\begin{aligned}
z_{1}^{g}(k) & \equiv r_{x}^{i}(k)-r_{x}^{g}(k) \\
& =\delta r_{x}+n_{1}^{g}(k), \\
z_{2}^{g}(k) & \equiv r_{y}^{i}(k)-r_{y}^{g}(k) \\
& =\delta r_{y}+n_{2}^{g}(k), \\
z_{3}^{g}(k) & \equiv h^{i}(k)-h^{g}(k) \\
& =\delta h+n_{3}^{g}(k) .
\end{aligned}
$$

Similarly to the above derivation, the measurements for velocity errors can be modeled as

$$
\begin{aligned}
z_{4}^{g}(k) & \equiv v_{x}^{i}(k)-v_{x}^{g}(k) \\
& =\delta v_{x}+n_{4}^{g}(k), \\
z_{5}^{g}(k) & \equiv v_{y}^{i}(k)-v_{y}^{g}(k) \\
& =\delta v_{y}+n_{5}^{g}(k), \\
z_{6}^{g}(k) & \equiv v_{z}^{i}(k)-v_{z}^{g}(k) \\
& =\delta v_{z}+n_{6}^{g}(k) .
\end{aligned}
$$

Thus the measurement Eqs. (33) and (34) can be expressed by

$$
\boldsymbol{z}^{g}(k)=H x(k)+\boldsymbol{n}^{g}(k),
$$

where

$$
\begin{aligned}
& \boldsymbol{z}^{g}(k)=\left[z_{1}^{g}(k), z_{2}^{g}(k), \ldots, z_{6}^{g}(k)\right]^{\mathrm{T}}, \\
& \boldsymbol{n}^{g}(k)=\left[n_{1}^{g}(k), n_{2}^{g}(k), \ldots, n_{6}^{g}(k)\right]^{\mathrm{T}} \\
& H \equiv\left[\begin{array}{llllllllll|l}
1 & 0 & 0 & 0 & 0 & 0 & 0 & 0 & 0 & 0 \\
0 & 1 & 0 & 0 & 0 & 0 & 0 & 0 & 0 & 0 \\
0 & 0 & 0 & 0 & 0 & 0 & 0 & 0 & 1 & 0 & \\
0 & 0 & 1 & 0 & 0 & 0 & 0 & 0 & 0 & 0 & 0_{6 \times 6} \\
0 & 0 & 0 & 0 & 1 & 0 & 0 & 0 & 0 & 0 & \\
0 & 0 & 0 & 0 & 0 & 0 & 0 & 0 & 0 & 1
\end{array}\right] .
\end{aligned}
$$

5 Nonlinear Filtering

\section{1 method 1}

First method of nonlinear filtering applied to INS/DGPS integration system is called "particle filtering (PF)" [2]. The main feature of PF is that it determines the conditional expectation of the variable to be estimated, with respect to the measurements, through a suitable random particle exploration of the weights of the particles.

The weights of particles are determined by the following likelihood ratio $Y\left(\mathcal{X}_{k}, \mathcal{Z}_{k}\right)$ based on the hypotheses

$$
\begin{aligned}
& \mathcal{H}_{0}: z^{g}(t)=n^{g}(t), \\
& \mathcal{H}_{1}: z^{g}(t)=H x(t)+n^{g}(t),
\end{aligned}
$$

$$
(t=1, \ldots, k) .
$$

Then, in the case of a Gaussian observation process, we have

$$
\begin{aligned}
Y\left(\mathcal{X}_{k}, \mathcal{Z}_{k}\right) & =\frac{p\left(\boldsymbol{z}^{g}(1), \ldots, \boldsymbol{z}^{g}(k) \mid \boldsymbol{x}(1), \ldots, \boldsymbol{x}(k)\right)}{p_{0}\left(\boldsymbol{z}^{g}(1), \ldots, \boldsymbol{z}^{g}(k) \mid \boldsymbol{x}(1), \ldots, \boldsymbol{x}(k)\right)} \\
& =\frac{\exp \left\{-\frac{1}{2} \sum_{t=1}^{k}\left\|\boldsymbol{z}^{g}(t)-H \boldsymbol{x}(t)\right\|_{R^{-1}(t)}^{2}\right\}}{\exp \left\{-\frac{1}{2} \sum_{t=1}^{k}\left\|\boldsymbol{z}^{g}(t)\right\|_{R^{-1}(t)}^{2}\right\}},
\end{aligned}
$$

where

$\mathcal{X}_{k}=\{\boldsymbol{x}(1), \ldots, \boldsymbol{x}(k)\}, \mathcal{Z}_{k}=\left\{\boldsymbol{z}^{g}(1), \ldots, \boldsymbol{z}^{g}(k)\right\}$. From Eqs.(36) - (38), we have

$$
\frac{E_{0}\left[x(k) Y\left(\mathcal{X}_{k}, \mathcal{Z}_{k}\right) \mid \mathcal{Z}_{k}\right]}{E_{0}\left[Y\left(\mathcal{X}_{k}, \mathcal{Z}_{k}\right) \mid \mathcal{Z}_{k}\right]}=E\left[x(k) \mid \mathcal{Z}_{k}\right]
$$

Thus by the low of large number

$$
\begin{aligned}
\frac{1}{N} \sum_{i=1}^{N} U_{k}^{i, N} x^{i, N}(k) \stackrel{N \rightarrow \infty}{P} E_{0}\left[U_{k} x(k) \mid \mathcal{Z}_{k}\right] \\
=E\left[x(k) \mid \mathcal{Z}_{k}\right]
\end{aligned}
$$

where $U_{k}^{i, N}$ is

$$
\begin{gathered}
U_{k}^{i, N}=\frac{Y\left(\mathcal{X}_{k}^{i, N}, \mathcal{Z}_{k}\right)}{\frac{1}{N} \sum_{j=1}^{N} Y\left(\mathcal{X}_{k}^{j, N}, \mathcal{Z}_{k}\right)} \\
\stackrel{N \rightarrow \infty}{P} \frac{Y\left(\mathcal{X}_{k}^{i, N}, \mathcal{Z}_{k}\right)}{E_{0}\left[Y\left(\mathcal{X}_{k}, \mathcal{Z}_{k}\right) \mid \mathcal{Z}_{k}\right]},
\end{gathered}
$$

" $i$ " indicates the $i$-th particle in $N$ particles. From Eq.(40), (41) the conditional expectation can be evaluted approximately.

$$
\begin{aligned}
& \frac{1}{N} \sum_{i=1}^{N} U_{k}^{i, N} x^{i, N}(k)=\sum_{i=1}^{N} p_{k}^{i, N} x^{i, N}(k) \\
& \stackrel{N \rightarrow \infty}{P} E\left[x(k) \mid \mathcal{Z}_{k}\right] .
\end{aligned}
$$


where the whight $p_{k}^{i, N}$ is given by

$$
p_{k}^{i, N}=\frac{Y\left(\mathcal{X}_{k}^{i, N}, \mathcal{Z}_{k}\right)}{\sum_{j=1}^{N} Y\left(\mathcal{X}_{k}^{j, N}, \mathcal{Z}_{k}\right)} .
$$

The PF algorithm may be summarized as follows:

In our DGPS/INS integration system, the state is defined by

$$
\bar{x}(k)=x(k)-\hat{x}(k),
$$

where $\hat{\boldsymbol{x}}(k)=E\left[\boldsymbol{x}(k) \mid \mathcal{Z}_{k}\right]$. Therefore we can summarize our PF algorithm as follows.

1) Generating the initial particle $\overline{\boldsymbol{x}}^{i, N}(0)$ according to the probability density $P_{\overline{\mathbf{x}}}(\bar{x}(0))$ with the equal weight $p_{0}^{i, N}=\frac{1}{N}$. Set $\mathrm{k}=0$.

2) Generate random number $w^{i, N}(k)$ and move the particle $\overline{\boldsymbol{x}}^{i, N}(k)$ to $\overline{\boldsymbol{x}}^{i, N}(k+1)$ according to Eq.(28) with $\boldsymbol{w}(k)=\boldsymbol{w}^{i, N}(k)$.

3) Compute the weight $p_{k+1}^{i, N}$ in Eq.(43) using the relation in Eq.(38).

4) Compute

$$
\hat{\mathfrak{x}}(k+1)=\sum_{i=1}^{N} p_{k+1}^{i, N} \overline{\mathfrak{x}}^{i, N}(k+1)
$$

and

$$
\overline{\boldsymbol{x}}^{i, N}(k+1)=\overline{\boldsymbol{x}}^{i, N}(k+1)-\hat{\mathfrak{x}}(k+1) .
$$

5) Repeat the steps 2) - 3).

\section{2 method 2}

Second method of nonlinear filtering [3] computes the likelihood $\alpha^{i, N}(k)$ of the particle $x^{i, N}(k)$ based on the observation $\boldsymbol{z}^{g}(k)$. Namely

$$
\begin{aligned}
\alpha^{i, N}(k) & =p\left(\boldsymbol{z}^{g}(k) \mid x^{i, N}(k)\right) \\
& =r\left(G\left(\boldsymbol{z}^{g}(k), \boldsymbol{x}^{i, N}(k)\right)\right)\left|\frac{\partial G}{\partial \boldsymbol{z}^{g}(k)}\right|,
\end{aligned}
$$

where

$$
G\left(\boldsymbol{z}^{g}(k), \boldsymbol{x}^{i, N}(k)\right)=\boldsymbol{z}^{g}(k)-H \boldsymbol{x}^{i, N}(k),
$$

and $r$ is the probability density of the observation noise $\boldsymbol{n}^{g}(k)$. Then, given $\boldsymbol{z}^{g}(k)$, the posterior probability is given by

$$
\begin{aligned}
P\left(x(k)=x^{i, N}(k) \mid \mathcal{Z}_{k}\right) & =\frac{\alpha^{i, N}(k) \frac{1}{N}}{\sum_{i=1}^{N} \alpha^{i, N}(k) \frac{1}{N}} \\
& =\frac{\alpha^{i, N}(k)}{\sum_{i=1}^{N} \alpha^{i, N}(k)} .
\end{aligned}
$$

Next, $N$ particles $\tilde{\boldsymbol{x}}^{1, N}(k), \ldots, \tilde{\boldsymbol{x}}^{N, N}(k)$ are obtained by resampling [3] of $\boldsymbol{x}^{1, N}(k), \ldots, \boldsymbol{x}^{N, N}(k)$ with probabilities

$$
P\left(\tilde{\boldsymbol{x}}^{i, N}(k)=\boldsymbol{x}^{i, N}(k) \mid \mathcal{Z}_{k}\right)=\frac{\alpha^{i, N}(k)}{\sum_{i=1}^{N} \alpha^{i, N}(k)} .
$$

By the resampling, each weight of $\tilde{x}^{1, N}(k), \ldots$, $\tilde{\boldsymbol{x}}^{N, N}(k)$ is $\frac{1}{N}$. The algorithm may be summarized as follows:

The steps 1) - 2) are the same as PF algorithm.

3) Compute $\alpha^{i, N}(k+1)$ from Eq.(47).

4) Generate $\tilde{\boldsymbol{x}}^{1, N}(k+1), \ldots, \tilde{\boldsymbol{x}}^{N, N}(k+1)$, by the resampling of $\overline{\boldsymbol{x}}^{1, N}(k+1), \ldots, \overline{\boldsymbol{x}}^{N, N}(k+1)$.

5) Repeat the steps 2) - 4).

\section{Conclusion}

In this paper, we have derived two nonlinear filtering methods for land vehicle In-Motion Alignment by integrating INS and DGPS. Applying our algorithms to the real system, we should consider the computational time for their real time implimentations.

\section{References}

[1] R. M. Rogers: Large Azimuth INS Error Models for In-Motion Alignment Land-Vehicle Positioning, Proceedings of The Institute of Navigation, National Technical Meeting 2001, Longbeach, CA, January, 2001.

[2] H.Caravalho, P. D. Moral, A.Monin and G. Salut: Optimal nonlinear filtering in GPS/INS integration, IEEE Trans. on Aerospace and Electoronic Systems, Vol.33, No.3,pp. 835-850(1997)

[3] G. Kitagawa Monte Carlo Filter and Smoother for Non-Gaussian Nonlinear State Space Models, Jounal of Computational and Graphical Statistics, Vol. 5, pp1-25, 1996.

[4] Will Gersch, Genshiro Kitagawa: General State Space Modeling in Statistical Methods in Control and Signal Processing, T. Katayama and S. Sugimoto, eds., Marcel Dekker, 1997, pp. 37-81. 